\title{
(2) OPEN ACCESS \\ National approaches to trichiasis surgical follow-up, outcome assessment and surgeon audit in trachoma-endemic countries in Africa
}

\author{
Grace Mwangi 우, ${ }^{1}$ Paul Courtright, ${ }^{2}$ Anthony W Solomon (i) $^{3}$
}

\begin{abstract}
- Supplemental material is published online only. To view please visit the journal online (http://dx.doi.org/10.1136/ bjophthalmol-2019-315777).

${ }^{1}$ Department of Surgery, Division of Ophthalmology, University of Cape Town, Cape Town, South Africa

${ }^{2}$ Kilimanjaro Centre for Community Ophthalmology, Division of Ophthalmology, University of Cape Town, Observatory, South Africa ${ }^{3}$ Department of Control of Neglected Tropical Diseases, World Health Organization, Geneva, Switzerland
\end{abstract}

\section{Correspondence to}

Grace Mwangi, Department of Surgery, Division of Ophthalmology, University of Cape Town, Cape Town, South Africa; grace.mwangi@alumni.uct.ac.za

Received 14 February 2020 Revised 2 April 2020 Accepted 25 June 2020 Published Online First 26 July 2020

\author{
ABSTRACT \\ Background Poor outcomes of trichiasis surgery, \\ including postoperative trichiasis, are common in many \\ trachoma-endemic countries in Africa. To improve \\ outcomes, WHO recommends regular follow-up and \\ outcome assessment of surgical cases plus audit of \\ trichiasis surgeons.
}

Aims To assess national approaches to trichiasis surgical follow-up, outcome assessment and audit, and identify national targets for good surgical outcome (defined as the percentage of patients undergoing surgery for trichiasis remaining free of post-operative trichiasis for a defined interval after surgery).

Methods A cross-sectional survey was carried out between May and July 2018, involving all 29 knowntrachoma-endemic countries in Africa. An emailed questionnaire was used to collect information on nationa targets for surgical outcomes, policies, monitoring and strategies to address underperformance by surgeons.

Results All national programmes provided information; 2 of the 29 had not yet implemented trichiasis surgery as part of their trachoma elimination programme. Findings from 27 countries are therefore reported. Only four countries reported having a national policy for trichiasis surgery follow-up and outcome assessment and only two had a national policy for conducting audits of trichiasis surgeons. Only 9 of the 27 countries had a cut-off point at which poorly performing surgeons would be instructed to discontinue surgery until retraining or other interventions had been undertaken.

Discussion To address the challenge of post-operative trichiasis and other poor outcomes, national trachoma programmes should create and implement policies and systems to follow up patients, assess surgical outcomes and monitor the performance of individual surgeons through post-surgical audits.

\section{INTRODUCTION}

Preliminary analyses of population-based data from the Global Trachoma Mapping Project ${ }^{1}$ indicate that, varying by location, $10-75 \%$ of people who currently have trichiasis have already had trichiasis surgery on at least one affected eyelid. ${ }^{2}$ The factors driving the occurrence of post-operative trachomatous trichiasis (PTT) cannot be ascertained from cross-sectional survey data, but these data suggest that in some settings, a considerable proportion of trichiasis surgeries that need to be undertaken are revision procedures. Quality assurance procedures for trichiasis surgery should be reviewed to ensure that outcomes of primary procedures can be maximised.

WHO recommends regular follow-up of patients and programmatic monitoring of trichiasis surgery outcomes to help reduce the incidence of PTT and to facilitate its management where it is detected. ${ }^{2} \mathrm{WHO}$ advises $^{3}$ that all operated trichiasis patients be seen on the first post-operative day for eye patch removal and examination of the wound. Further follow-up at 8-14 days is recommended; this is essential if nonabsorbable sutures are used. ${ }^{2}$ All patients should be followed up again 3-6 months after surgery. ${ }^{2}$ The primary purpose of each of these reviews is to allow the surgeon to assess the status of the operated eyelid(s) and make decisions, with the patient, regarding further management.

Parallel activities to improve outcomes of trichiasis surgery include supportive supervision and audit of trichiasis surgeons. The goal of an audit is to determine if individual surgeons have acceptable outcomes, need additional training and supervision or should discontinue providing surgery altogether. According to the Second Global Scientific Meeting on Trachomatous Trichiasis, ${ }^{2}$ for audit purposes, programmes should assess a sample of patients operated on by each individual practising surgeon. Data from an audit should be used to review the performance of new trichiasis surgeons 3-6 months after first certification, and of existing trichiasis surgeons as indicated. ${ }^{2}$ If a surgeon is found to have an unacceptably high incidence of PTT or other poor outcomes, retraining and more intensive supportive supervision by a more experienced surgeon is recommended. ${ }^{4-6}$

It is recognised that trichiasis patient follow-up, monitoring of outcomes and auditing of surgeons' performance are challenging for trachoma programmes. $^{2}$ Recommended policies and guidance on all aspects of trichiasis surgical service delivery have been developed by WHO and the International Coalition for Trachoma Control (ICTC), but adaption and adoption of these instruments at national level has been incomplete. There are structural and financial implications of implementation, which is made particularly difficult by the field environment: trachoma generally affects the poorest and most marginalised members of society, who often live in areas distant from health facilities. ${ }^{7}$ Such patients are often lost to follow-up after surgery, rendering post-operative care and monitoring of outcomes impossible. ${ }^{8}$ 
We undertook a survey to assess the availability of national policies and strategies for monitoring outcomes and for conducting surgical audits, and strategies for addressing poor surgical quality among surgeons in the 29 African countries where trachoma was still considered a public health problem in 2018.

\section{MATERIALS AND METHODS}

The survey was conducted in 29 trachoma-endemic countries in Africa (figure 1). Each national trachoma programme coordinator (or the equivalent) in these countries was invited to participate. Participants were recruited via email and requested to provide informed consent before completing a questionnaire. Ethical approval was obtained from the University of Cape Town Health Sciences Research Committee and the WHO Ethics Review Committee (0003034).

A single cross-sectional survey was carried out between April and July 2018. An emailed questionnaire (see online supplemen tary material) was used to collect information. Prior to formal use, the questionnaire was piloted to assess wording, content and format, with pilot respondents being one national trachoma programme coordinator and one ophthalmologist with extensive knowledge and experience in trachoma programmes. Feedback

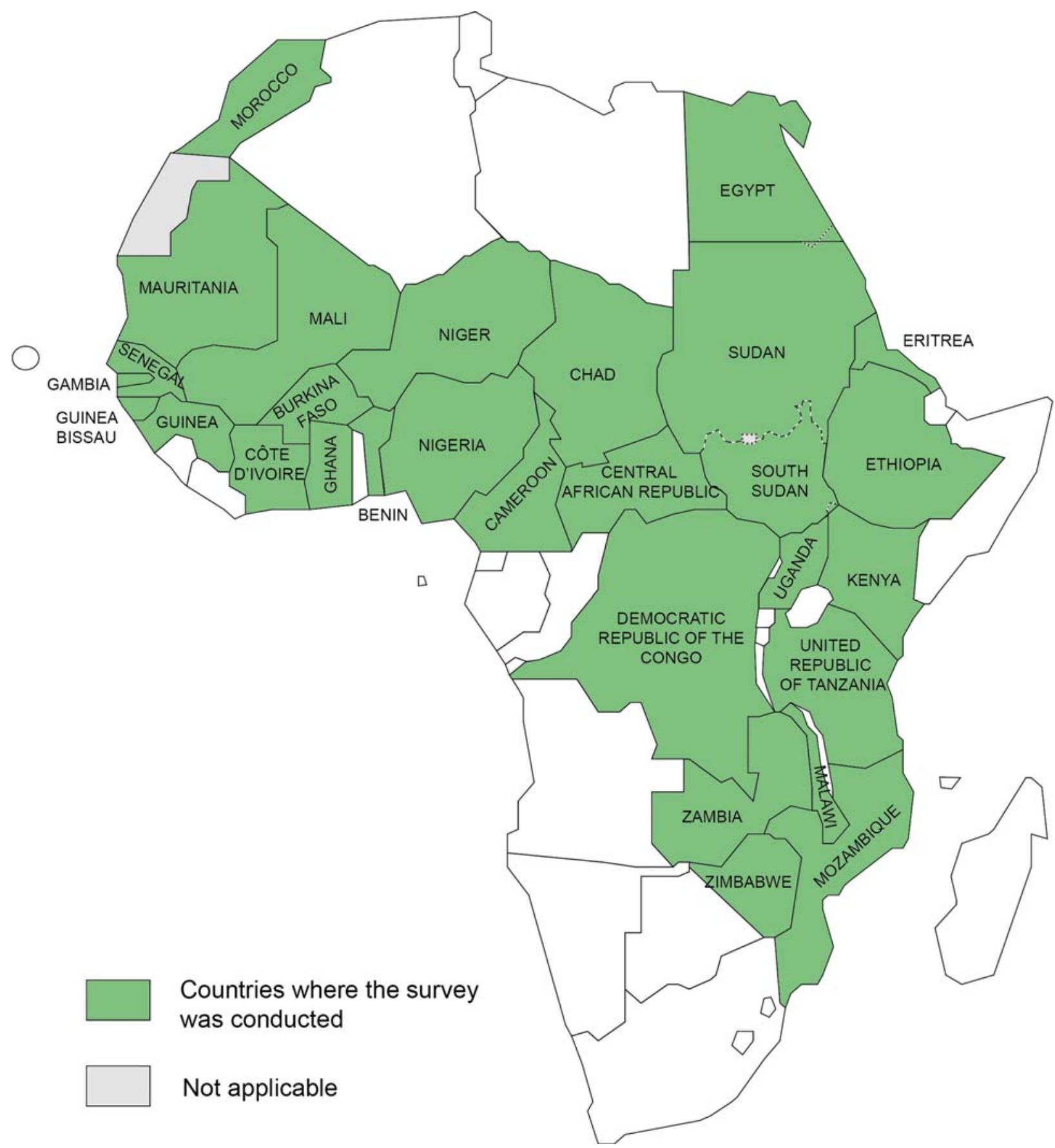

Figure 1 The 29 countries in Africa in which the survey was conducted. The boundaries and names shown and the designations used on this map do not imply the expression of any opinion whatsoever on the part of the World Health Organization concerning the legal status of any country, territory, city or area or of its authorities, or concerning the delimitation of its frontiers or boundaries. Dotted lines on maps represent approximate border lines for which there may not yet be full agreement. Credit: Illustrated by Alexei Mikhailov. 
from this pretest was used to refine the questionnaire, which was then translated into French and Portuguese. Further validation through pilot testing was undertaken with intended respondents who were bilingual in either English and French or English and Portuguese, to ensure that the translated instruments were equivalent to the English original.

Survey responses were entered into Microsoft Excel, checked for completeness and verified against the original response forms. Responses in French and Portuguese were translated into English and checked (through back-translation) for correctness. Aggregated tables were generated in Excel. Qualitative data were analysed using thematic analysis and narrative synthesis.

\section{RESULTS}

All 29 eligible national coordinators consented and participated. Two of 29 trachoma-endemic countries had not yet commenced implementation of a trichiasis surgery programme; both were excluded from data analysis. Findings are presented for 27 countries.

Only 4 (15\%) of 27 countries reported having a national policy for follow-up of trichiasis patients after surgery (figure 2). Of the remaining 23, 11 did not have national policies or guidelines but reported that systems and structures for follow-up of patients after surgery were nevertheless in place; 10 countries had adopted the ICTC preferred practice guidelines ${ }^{8-10}$; and two countries had no policy or agreed on follow-up procedure for outcome assessment.

Sixteen $(59 \%)$ of 27 countries reported mandating all three recommended follow-up points: 1 day after surgery, 1-2 weeks after surgery, and 3-6 months (or 1 year) after surgery. Of the remaining 11 countries, 4 countries mandated at least two followup points, 6 countries had only one mandated follow-up point, and 1 country did not have any mandated follow-up points.

Three countries had not set a national target for good surgical outcomes (defined as the percentage of patients undergoing trichiasis surgery remaining free of PTT at 3-6 months or 1 year). Among the remaining 24 countries, 3 reported having a target of $100 \%$ good outcomes, 14 countries had a target of $90 \%, 1$ country had a target of $85 \%$, and 6 countries had a target of $80 \%$.

Only two countries reported having a national policy regarding surgeon audits while 19 countries $(70 \%)$ had no specific national surgeon audit policy but reported that audits of trichiasis surgeons were undertaken by supervisors $(n=14)$ or had adopted other guidelines, such as the Queen Elizabeth Diamond Jubilee Trust funded surgical audit guidelines $(n=5)$ (figure 3$)$. These guidelines recommend the use of surgical audit as part of quality assurance to improve the quality of trichiasis surgeries delivered. ${ }^{11}$ The remaining six countries had neither a national policy nor an agreed approach to conducting audits of surgeons.

It is recommended that surgeons with poor outcomes (with the threshold for 'poor' being defined locally) should undergo a retraining and certification process. ${ }^{11}$ In this survey, only 10 (37\%) of 27 countries reported having a nationally accepted threshold above which a surgeon should discontinue surgery. Variable thresholds were reported among those 10 countries; $30 \%$ poor outcomes ( 3 countries), 25\% (1 country), 20\% (4 countries), 15\% (1 country) and 10\% (1 country).

Of the 17 countries that reported having national targets for good outcomes ( $\leq 10 \%$ PTT), only 1 had a nationally defined threshold of $10 \%$ at which a surgeon should discontinue surgery until retraining or other interventions have been undertaken. Six countries reported that a surgeon-level incidence of 15-30\% PTT would prompt this action, while 10 countries had no PTT threshold for surgeons to discontinue surgery.

Countries recommended different approaches to manage PTT. In 19 (70\%) of 27 countries, cases of PTT or other complications were reportedly managed by another trichiasis surgeon who was more experienced than the original surgeon $(n=13)$ or by a specially trained ophthalmologist $(n=6)$. Seven countries reported that surgery for PTT was done by the same surgeon
No policy but has an agreed procedure for
outcome assessment

No policy but has adopted ICTC preferred practice guidelines

\section{Has a national policy for follow up}

\section{No policy and no agreed follow procedure for outcome assessment}

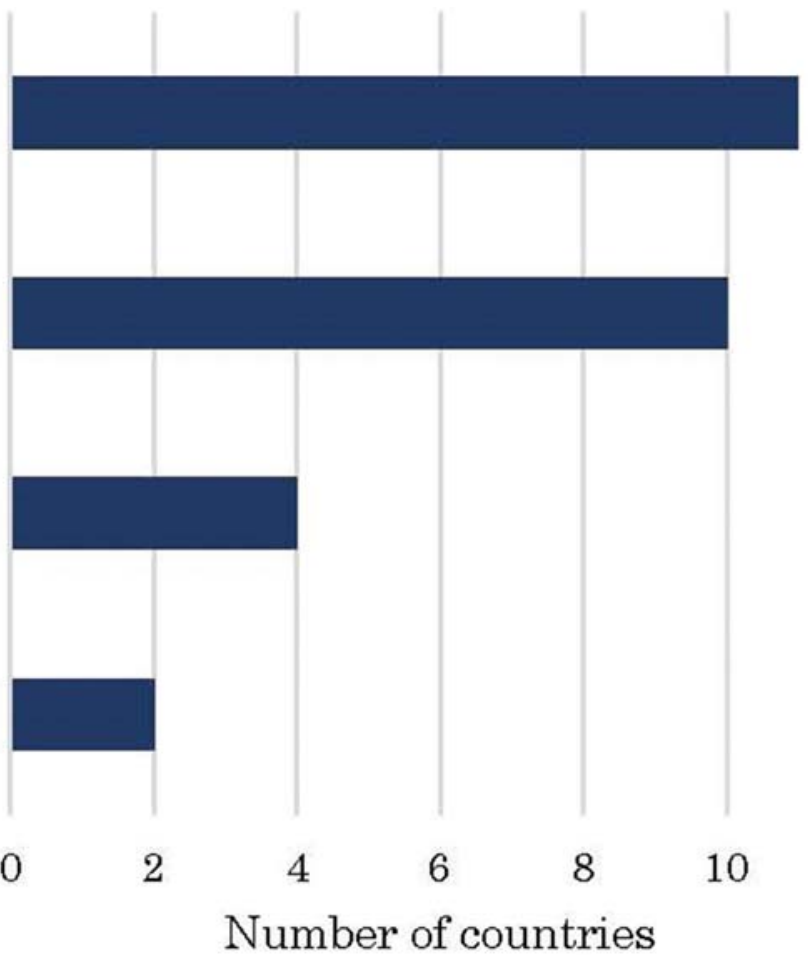

Figure 2 Availability of national policies for trichiasis surgery follow-up. 


\section{No policy but audits of trichiasis surgeons are undertaken by supervisors}

\author{
No policy and no agreed approach to \\ conducting audits
}

\author{
No policy but the country has adopted other \\ guidelines (Trust-funded guidelines)
}

\section{Has a policy for conducting surgical audits}

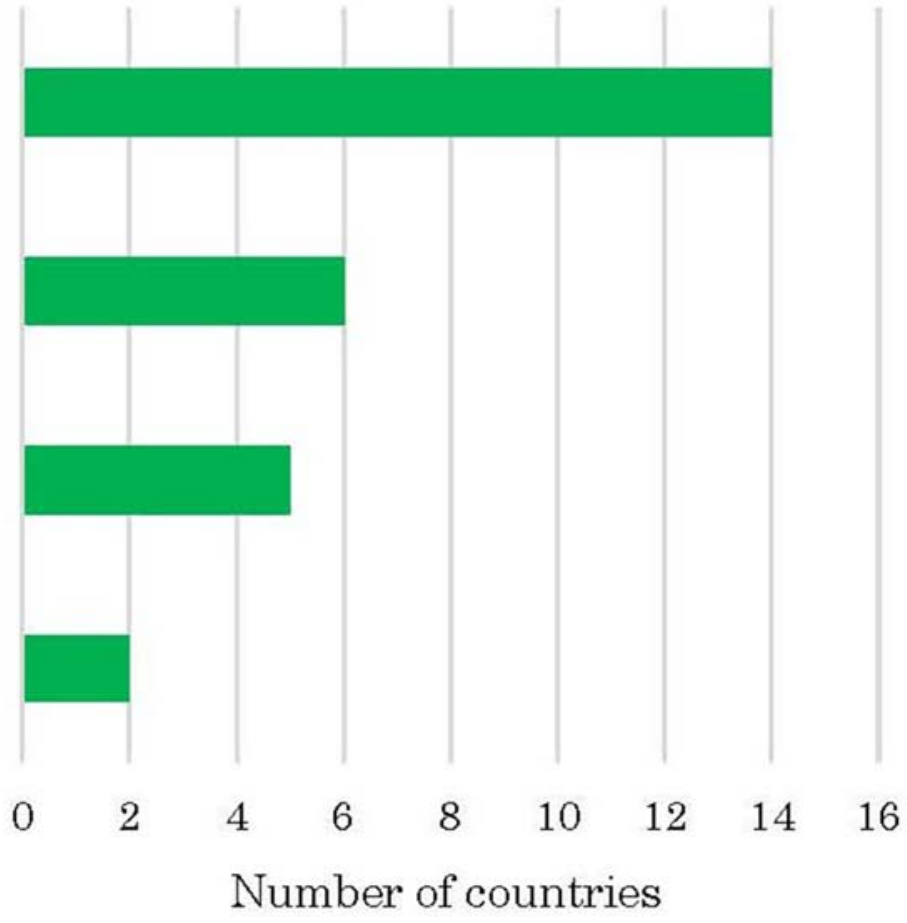

Figure 3 Countries with a national policy for conducting surgical audits.

who had performed the first procedure. One country did not specify its approach for managing PTT.

Fifteen $(88 \%)$ of the 17 countries that reported aiming for $\leq 10 \%$ PTT also reported that cases of PTT were either managed by a specially trained ophthalmologist or a more experienced trichiasis surgeon. The remaining two countries reported that post-operative cases were either managed by the surgeon who performed the first procedure or the surgeon who identified them during a surgical campaign.

Some key challenges highlighted by national coordinators included (1) lack of funds to support the full range of trichiasisrelated activities; (2) limited national data to inform planning; (3) limited numbers of secondary eye care units for post-operative management; (4) lack of proper follow-up systems for trichiasis patients after surgery; (5) weak referral systems; (6) refusal by patients to undergo additional surgery; and (7) shortage of trained human resources to manage PTT.

\section{DISCUSSION}

In 2010, the third Global Scientific Meeting on Trachoma recommended that national programmes should routinely report outcomes of trichiasis surgery, with a target of achieving $\leq 10 \%$ PTT at 1 year after surgery. ${ }^{12}$ In November 2018, the fourth Global Scientific Meeting on Trachoma revised that recommendation, requesting that cases be divided into those that had minor vs major trichiasis (see below) pre-operatively, and suggesting that the timing of the final follow-up point be 3-6 months after surgery. For minor trichiasis ( $\leq 5$ upper eyelid eyelashes touching the eyeball), the target was set as $<10 \%$ PTT at the final follow-up point, while for major trichiasis ( $>5$ upper eyelid eyelashes touching the eyeball), it was set as $<20 \%$. $^{13}$

The work reported in this paper indicates that in 2018, prior to the change noted earlier, only half the trachoma-endemic countries in Africa had set national outcome targets consistent with the then-current (2010) WHO recommendation (ie, >90\% good outcomes). We have recently reviewed the published literature and found that even in the often highly controlled confines of the research setting, a PTT incidence of $<20 \%$ at 3-12 months after surgery is rarely achieved. ${ }^{14}$ Without the combined framework of policies and other health system elements to support the practice of follow-up and outcome assessment, however, not only is the incidence of poor outcomes likely to remain high, it will continue to be unrecorded and unremarked.

We therefore note with concern that in 2018 , only $42 \%$ of countries in Africa reported having their own specific national policy for follow-up of trichiasis patients after surgery $(15 \%)$ or having adopted the ICTC preferred practice guidelines ${ }^{8}$ (27\%). Only $59 \%$ of countries expected surgeons to see patients at all three WHO-recommended follow-up points. Another 15\% of countries promoted at least two follow-up periods: 1 day and 3-6 months (or 1 year) after surgery. Of the seven countries that did not have the second recommended follow-up (1-2 weeks), only four reported routinely supplying and using absorbable sutures which might have made that follow-up less important. The remaining three countries provide non-absorbable sutures which should be removed at 7-10 days. This may mean that many patients in these countries retain unremoved sutures long after surgery. Although studies have found that the use of absorbable sutures does not lower the risk of PTT, ${ }^{15}$ retained sutures can cause granulomata, promote infection and traumatise the cornea. ${ }^{16}$ Trachoma elimination programmes should consider using absorbable sutures in case patients miss the 7-10-day follow-up for suture removal.

Surgical audit provides a crucial link to ensure that policy is translated into individual practice and good outcomes. As an independent assessment of surgical outcomes achieved by a specific surgeon, audit is an important quality assurance tool for national programmes and should be part of ongoing supportive supervision. Unfortunately, our survey indicated that in 2018, audits were given inadequate attention in most trachomaendemic countries in Africa. The lack of identification of a cut-off for determining which surgeons need retraining suggests that most countries were not using audits for decision-making. The 
implication is that surgeons with unacceptably high incidence rates of PTT might continue to operate. Even in countries that had programme-level targets for incidence of PTT, a minority had surgeon-level thresholds prompting action for discontinuation or retraining, suggesting a disconnect between the targets and the policies in place to help achieve them. It would be wrong, however, to focus exclusively on the capacity of audit to identify providers who are performing sub-optimally: supervision and audit with feedback improve general health worker performance, could encourage higher surgical productivity ${ }^{17}$ and might reduce the current excessive surgeon attrition rate. ${ }^{18} 19$

We acknowledge several limitations of our study. First, we relied on self-reports from national coordinators. Second, we collected information at national level in 29 countries, rather than on actual practice regarding the management of trichiasis and PTT. It is likely that the picture painted by national coordinators is an over-simplification of the true situation on the ground, where practices could well vary between different subnational administrative areas. National policies, however, are important drivers of local action. Third, our definition of 'good surgical outcome' was essentially limited to an absence of PTT. Other unfavourable outcomes of trichiasis surgery, such as granuloma or eyelid contour abnormality with or without eyelid closure defects may, unfortunately, also be common. ${ }^{20}$ It is possible (but by no means certain) that national policies and approaches for assessment of PTT will also help in monitoring for these other complications.

Availability of reliable, quality-assured and quality-controlled data on the prevalence of active trachoma and trichiasis ${ }^{2122}$ have been key to an evidence-based expansion in work against trachoma in recent years. ${ }^{23}$ Surgical follow-up data are amenable to similar standardisation and quality-marking. ${ }^{24}$ The recent infusion of financial and technical support for trichiasis throughout Africa should, through concerted effort of governments and their partners, make the requisite improvements possible. Specific attention is needed now to ensure that programmes generate the appropriate data on trichiasis surgery outcomes and use them to drive better results for patients.

Correction notice This paper has been corrected since it was published online. Figure 1 has been replaced.

Twitter@anthonywsolomon and @courtright_paul.

Acknowledgements We thank the national trachoma programme coordinators who participated in this study. We are also grateful to Alexei Mikhailov for producing Figure 1.

Contributors GM, PC and AWS contributed to the design and implementation of the study, analysis of the results and writing of the manuscript.

Funding This study was part of a Master of Public Health course funded by the Queen Elizabeth Diamond Jubilee Trust.

Disclaimer The authors alone are responsible for the views expressed in this article and they do not necessarily represent the views, decisions or policies of the institutions with which they are affiliated.

Competing interests None declared.

Provenance and peer review Not commissioned; externally peer reviewed. Data availability statement All data relevant to the study are included in the article or uploaded as supplementary information.

Open access This is an open access article distributed under the terms of the Creative Commons Attribution N-Noncommercial IGO License (https://creativecommons.org/ licenses/by/3.0/igo/), which permits use, distribution, and reproduction for non- commercial purposes in any medium, provided the original work is properly cited. In any reproduction of this article there should not be any suggestion that WHO or this article endorse any specific organization or products. The use of the WHO logo is not permitted. This notice should be preserved along with the article's original URL.

\section{ORCID iDs}

Grace Mwangi http://orcid.org/0000-0003-2082-2098

Anthony W Solomon http://orcid.org/0000-0001-7101-6649

\section{REFERENCES}

1 Solomon AW, Kurylo E. The global trachoma mapping project. Community Eye Health 2014:27:18.

2 World Health Organization. World Health Organization Alliance for the Global Elimination of Trachoma by 2020: second global scientific meeting on trachomatous trichiasis: Cape Town, 4-6 November 2015. World Health Organization 2016.

3 Solomon AW, Zondervan M, Kuper $\mathrm{H}$, et al. Trachoma control: a guide for programme managers. Geneva: World Health Organization; 2006.

4 Gower EW, Kello AB, Kollmann KHM. Training trichiasis surgeons: ensuring quality. Community Eye Health 2014;27:58.

5 Courtright P, Burton M, Emerson P. Eliminating trichiasis: the next steps forward Community Eye Health 2012;25:38.

6 Buchan JC, Limburg H, Burton MJ. Quality assurance in trichiasis surgery: a methodology. Br J Ophthalmol 2011;95:331-4.

7 Bangert M, Molyneux DH, Lindsay SW, et al. The cross-cutting contribution of the end of neglected tropical diseases to the sustainable development goals. Infect Dis Poverty. 2017:6:73

8 International Coalition for Trachoma Control. Organizing trichiasis surgical outreach: a preferred practice for program managers in Ethiopia. April 2015

9 International Coalition for Trachoma Control. Training trichiasis surgeons for trachoma elimination programs. March 2018.

10 International Coalition for Trachoma Control. Supportive supervision for trachomatous trichiasis programmes. October 2016.

11 Sightsavers. A guide to conducting trachomatous trichiasis surgical audits for quality improvement. 2019. Working Draft version 03 Nov 16.

12 World Health Organization. Report of the 3rd Global Scientific Meeting on Trachoma, Johns Hopkins University, Baltimore, MA, 19-20 July 2010 (WHO/PBD/2.10). Geneva: World Health Organization; 2010.

13 World Health Organization. Report of the 4th Global Scientific Meeting on Trachoma, Geneva, 27-29 November 2018 (WHO/CDS/NTD/PCT/2019.03). Geneva: World Health Organization; 2019.

14 Mwangi G, Courtright P, Solomon AW. Post-operative trichiasis in Africa: a systematic review. [Submitted]. 2019.

15 Rajak SN, Habtamu E, Weiss HA, et al. Absorbable versus silk sutures for surgical treatment of trachomatous trichiasis in Ethiopia: a randomised controlled trial. PLoS Med 2011;8:12.

16 Merbs S, Resnikoff S, Kello AB, et al. Trichiasis surgery for trachoma, 2nd ed. Vol. 77. Geneva: World Health Organization, 2015: 81-3.

17 Lewallen S, Mahande M, Tharaney M, et al. Surgery for trachomatous trichiasis: findings from a survey of trichiasis surgeons in Tanzania. $\mathrm{Br} J$ Ophthalmol 2007:91:143-5.

18 Habtamu E, Rajak SN, Gebre T, et al. Clearing the backlog: trichiasis surgeon retention and productivity in Northern Ethiopia. Schachter J, editor. PLOS Negl Trop Dis 2011;5: e1014.

19 Rowe AK, De Savigny D, Lanata CF, et al. How can we achieve and maintain highquality performance of health workers in low-resource settings? Lancet 2005;366:1026-35.

20 Gower EW, Merbs SL, Munoz BE, et al. Rates and risk factors for unfavorable outcomes 6 weeks after trichiasis surgery. Investig Ophthalmo/ Vis Sci 2011;52:2704-11.

21 Solomon AW, Pavluck AL, Courtright P, et al. The global trachoma mapping project: methodology of a 34-country population-based study. Ophthalmic Epidemiol 2015:22:214-25

22 Solomon AW, Willis R, Pavluck AL, et al. Quality assurance and quality control in the global trachoma mapping project. Am J Trop Med Hyg 2018;99:858-63.

23 Courtright P, Rotondo LA, MacArthur C, et al. Strengthening the links between mapping, planning and global engagement for disease elimination: lessons learnt from trachoma. Br J Ophthalmol 2018:102:1324-7.

24 World Health Organization. Informal consultation on a tracking system for patients with trachomatous trichiasis. Rollins School of Public Health, Emory University, Atlanta, USA, Wednesday 30 September 2015 (WHO/HTM/NTD/2016.1). Geneva: World Health Organization. World Health Organization; 2017, 2016. 